\title{
Anti-metastatic effects of DNA vaccine encoding single-chain trimer composed of MHC I and vascular endothelial growth factor receptor 2 peptide
}

\author{
RUILING CHEN $^{1,2^{*}}$, SHENGCHAO WANG ${ }^{3 *}$, YUNLIANG YAO ${ }^{4}$, YUN ZHOU ${ }^{2}$, CHONG ZHANG $^{1}$, \\ JIE FANG $^{1}$, DAYONG ZHANG ${ }^{1}$, LIHUANG ZHANG ${ }^{1}$ and JIANPING PAN ${ }^{1}$
}

\begin{abstract}
${ }^{1}$ Department of Clinical Medicine, Zhejiang University City College School of Medicine, Hangzhou, Zhejiang 310015;
${ }^{2}$ Department of Pathogen Biology, Zhejiang University School of Medicine, Hangzhou, Zhejiang 310058 ; ${ }^{3}$ Section Three of the Department of General Surgery, First Affliated Hospital, Shihezi University School of Medicine, Shihezi, Xinjiang 832008; ${ }^{4}$ Department of Microbiology and Immunology, Huzhou Teacher's College School
\end{abstract} of Medicine, Huzhou, Zhejiang 313000, P.R. China

Received October 27, 2014; Accepted February 9, 2015

DOI: $10.3892 /$ or.2015.3820

\begin{abstract}
Vascular endothelial growth factor receptor 2 (VEGFR2)-mediated signaling is the key rate-limiting step in angiogenesis. VEGFR2 serves as the most important target of anti-angiogenic therapy for cancers. Single-chain trimer (SCT) comprising antigen peptide, $\beta 2$-microglobulin $(\beta 2 \mathrm{~m})$, and major histocompatibility complex (MHC) class I heavy chain was a particularly powerful strategy involved in the increase of the potency of DNA vaccine against tumors and infections. In the present study, we constructed an SCT-encoding VEGFR2 antigen peptide [aa400-408, also known as kinase insert domain-containing receptor (KDR2)], $\beta 2 \mathrm{~m}$, and mouse MHC class I heavy chain $\mathrm{H}-2 \mathrm{D}^{\mathrm{b}}$ [pcDNA3.1(+)-KDR2- $\beta 2 \mathrm{~m}-\mathrm{H}-2 \mathrm{D}^{\mathrm{b}}$, or SCT-KDR2]. The constructed SCT-KDR2 DNA was efficiently expressed in the human A293 embryonic kidney cell line. Intradermal immunization of C57BL/6 mice with SCT-KDR2 DNA was able to successfully break self-immunological tolerance and induce robust cytotoxic T-lymphocyte (CTL) response to VEGFR2, leading to marked suppression of tumor cell-induced angiogenesis and metastasis in murine models of B16 melanoma and 3LL Lewis lung carcinoma. Taken together, the results showed that VEGFR2-targeted SCT vaccination is an effective modality that can be utilized in anti-angiogenic active immunotherapy for various types of cancer.
\end{abstract}

Correspondence to: Professor Jianping Pan, Department of Clinical Medicine, Zhejiang University City College School of Medicine, 50 Huzhou Street, Hangzhou, Zhejiang 310015, P.R. China

E-mail:.jppan@zucc.edu.cn

*Contributed equally

Key words: DNA vaccine, single-chain trimer, anti-angiogenic immunotherapy, VEGFR2

\section{Introduction}

The formation of new blood vessels that supply the tumor mass, or tumor angiogenesis, is a characteristic of cancer and is important in tumor outgrowth, progression and metastasis, due to the proliferation and metastasis of malignant tumors depending on the sufficient nutrition supplied by the new blood vessels (1-5). Molecules have been demonstrated as angiogenesis-promoting factors $(3,6-8)$, such as vascular endothelial growth factor (VEGF), epidermal growth factor (EGF), fibroblast growth factor (FGF), placental growth factor (PlGF), transforming growth factor- $\alpha / \beta$ (TGF- $\alpha / \beta)$, angiogenin, CD105 (endoglin), the anthrax-toxin-receptor (ATR, TEM8), prostate-specific membrane antigen (PSMA), connective tissue growth factor (CTGF), urokinase plasminogen activator (uPA) and the up-to-date reported galactin-1 (Gal1) (also known as galectin, a member of a conserved family of animal lectins). It has been demonstrated, however, that VEGF-mediated signaling through vascular endothelial growth factor receptor 2(VEGFR2) is the critical rate-limiting step in tumor angiogenesis, and is crucial in angiogenesis or neovascularization $(4,9)$.

VEGFR2, also known as kinase insert domain-containing receptor (KDR) in human and fetal liver kinase 1 (flk1) in mouse, is involved as a therapeutic target of anti-angiogenic therapy for various types of cancer because VEGFR2 is strictly expressed on endothelial cells and upregulated when these cells proliferate during angiogenesis in the tumor vasculature. A number of approaches have been previously used to block the VEGFR2-mediated signaling in antiangiogenic therapy for various types of cancer, including neutralization of VEGF by monoclonal antibodies (10-12), therapy with T4 phage displaying VEGFR2 (13), administration of small-molecule receptor tyrosine kinase inhibitors (RTKIs) $(6,14)$, and vaccination with endothelial cells (15), VEGFR2 cDNA transfected-bacteria $(16,17)$ or VEGFR2 protein (18) pulsed- or mRNA- (19) and cDNA- (20) transfected-dendritic cells (DC). Furthermore, it was identified 
that there were three $\mathrm{H}-2 \mathrm{D}^{\mathrm{b}}$-restricted $\mathrm{CD} 8^{+} \mathrm{T}$-cell epitopes in mouse VEGFR2, designated as KDR1, KDR2 and KDR3, respectively (21). KDR1 was derived from the intracellular domain of VEGFR2, while KDR2 and KDR3 were derived from the extracellular domain (21). Immunization with KDR2 or KDR3, but not KDR1 peptides, was able to break self-tolerance and induce a specific $\mathrm{CD} 8^{+}$cytotoxic T-lymphocyte (CTL) immune response in C57BL/6 mice, thereby inhibiting angiogenesis and tumor growth in a mouse model (21). In addition, it was found that KDR2 was more efficiently processed by proteasomes and/or transported to the endoplasmic reticulum by transporter-associated with antigen processing (TAP) rather than KDR3, offsetting the higher affinity of KDR3 (21). Therefore, KDR2 is an attractive candidate of $\mathrm{CD}^{+} \mathrm{T}$-cell epitopes suitable for active immunotherapy targeting tumor blood vessels in which the main aim is to break self-tolerance of the molecules that promote angiogenesis.

It has been demonstrated that the single-chain trimer (SCT) composed of major histocompatibility complex (MHC) class I heavy chain, $\beta 2$-microglobulin $(\beta 2 \mathrm{~m})$, and antigen peptide was a particularly powerful strategy used to increase the potency of DNA vaccine against tumor (22-26) and infection (22,27-31). In the present study, we constructed a DNA vaccine encoding an SCT composed of KDR2, $\beta 2 \mathrm{~m}$ and $\mathrm{H}-2 \mathrm{D}^{\mathrm{b}}$ [pcDNA3.1(+)KDR2- $\beta 2 \mathrm{~m}-\mathrm{H}-2 \mathrm{D}^{\mathrm{b}}$, or SCT-KDR2] and tested its ability to induce specific CTL response to VEGFR2 and its capacity to inhibit tumor-induced angiogenesis and metastasis in mouse models.

\section{Materials and methods}

Mice and cells. Female C57BL/6 mice $\left(\mathrm{H}-2^{\mathrm{b}}\right), 6-8$ weeks of age, were purchased from SLAC Laboratory Animal Co., Ltd., (Shanghai, China) and were housed in specific pathogen-free conditions. All the animal procedures were performed according to approved protocols and in accordance with recommendations for the proper use and care of laboratory animals by the Institutional Committee. B16 (a mouse melanoma cell line of C57BL/6 origin, H-2 ${ }^{b}$ ), EL-4 (a lymphoma cell line, H-2 ${ }^{\text {b }}$, A293 (a human embryonic kidney cell line), and 3LL (a Lewis lung carcinoma cell line, $\mathrm{H}-2^{\mathrm{b}}$ ) were obtained from the American Type Culture Collection (Manassas, VA, USA). H5V ( $\left.\mathrm{H}-2^{\mathrm{b}}\right)$, a mouse endothelial cell line expressing VEGFR2, was a kind gift from Dr A. Vecchi (Instituto Mario Negri, Via Eritrea, Milan, Italy) as indicated in our previous report (20). 3LL-sflk1 (3LL Lewis lung carcinoma cells stably expressing extracellular domain of VEGFR2) was established in our laboratory as previously described (20).

Plasmid DNA constructs and DNA preparation. The construction of pcDNA3.1(+)- KDR2- $\beta 2 \mathrm{~m}-\mathrm{H}-2 \mathrm{D}^{\mathrm{b}}$ (SCT-KDR2) and pcDNA3.1(+)-OVA- $\beta 2 \mathrm{~m}-\mathrm{H}-2 \mathrm{D}^{\mathrm{b}}$ (SCT-OVA) is shown in Fig. 1. To construct SCT-KDR2, an insert containing the mouse $\beta 2 \mathrm{~m}$ signal peptide plus $\mathrm{H}-2 \mathrm{D}^{\mathrm{b}}$-restricted immunodominant KDR2 (aa400-408) epitope and flanking NheI/SpeI restriction enzyme sites was created by annealing two single-stranded oligo-nucleotides [sense, 5'-CATGCTAGCATGGCTCGC TCGGTGACCCTAGTCTTTCTGGTGCTTGTCTCACTGA CCGGCTTGTATGCTGTCATCCTCACCAACCCCATTTC
AATGACTAGTGGTG and antisense, 5'-CACCACTAGTCA TTGAAATGGGGTTGGTGAGGATGACAGCATACAAGC CGGTCAGTGAGACAAGCACCAGAAAGACTAGGGTCA CCGAGCGAGCCATGCTAGCATG-3', Sangon Biotech (Shanghai) Co., Ltd., Shanghai, China]. The generated cDNA was linked, through the SpeI restriction site, with Linker 1 which was generated by annealing two single-stranded oligonucleotides flanking the SpeI and HindIII restriction sites as follows:5'-GACTAGTGGTGGCGGAGGTTCTGGTGGCGG AGGTTCTGGTGGCGGAGGTTCTGGTGGCGGAGGTTC TAAGCTTGGG-3' (sense) and 5'-CCCAAGCTTAGAACCT CCGCCACCAGAACCTCCGCCACCAGAACCTCCGCCA CCAGAACCTCCGCCACCACTAGTC-3' (antisense). The yielded cDNA was subcloned into pcDNA3.1(+) (Invitrogen, Carlsbad, CA, USA) between the NheI and HindIII restriction sites. cDNA encoding $\mathrm{H} 2-\mathrm{D}^{\mathrm{b}}$ and $\beta 2 \mathrm{~m}$ were cloned by RT-PCR from the spleen cells of C57/B6 mouse with a Superscript One-Step RT-PCR system (Invitrogen). Primers used for H-2D ${ }^{\text {b }}$ were: 5'-TTGCGGCCGCGGCCCACACTCGATGC GGT-3' (sense) and 5'-GCTCTAGATCACGCTTTACAATCT CGGAG-3' (antisense). Full-length H-2D ${ }^{\mathrm{b}}$ cDNA was then inserted into the above constructed recombinant vector between the Not I and XbaI restriction sites. Primers used for $\beta 2 \mathrm{~m}$ were: 5'-CCCAAGCTTATCCAGAAAACCCCTCAAA TTC-3' (sense) and 5'-CGGGATCCCATGTCTCGATCCCAG TAGAC-3' (antisense). The $\beta 2 \mathrm{~m}$ cDNA was subcloned into the recombinant plasmid between the HindIII and BamHI restriction sites. Linker 2 was produced by annealing the following two single-stranded oligo-nucleotides flanking the Bam HI and NotI restriction sites: 5'-CGCGGATCCGGTGGCGGAGGT TCTGGTGGCGGAGGTTCTGGTGGCGGAGGTTCTGGT GGCGGAGGTTCT-3' (sense) and 5'-TTGCGGCCGCAAGA ACCTCCGCCACCAGAACCTCCGCCACCAGAACCTCC GCCACCAGAACCTCCGCCACC-3' (antisense) (Sangon Biotech). cDNA encoding Linker 2 was subsequently inserted into the above generated recombinant vector to form the SCT-KDR2. To generate SCT-OVA, $\beta 2 \mathrm{~m}$ signal peptide plus immunodominant KDR2 (aa400-408) epitope was replaced by $\beta 2 \mathrm{~m}$ signal peptide plus $\mathrm{H}-2 \mathrm{D}^{\mathrm{b}}$-restricted immunodominant ovalbumin (OVA) epitope (aa257-265, ESIINFEKL) which was generated by annealing the synthesized oligo-nucleotides (sense, GCTAGCATGGCTCGCTCGGTGACCCTAGTCT TTCTGGTGCTTGTCTCACTGACCGGCTTGTATGCTTA GTTGTACAGTTCAGCTCTTTATAATACTAGTGGTG-3' and antisense, 5'-CACCACTAGTATTATAAAGAGCTGAA CTGTACAACTAAGCATACAAGCCGGTCAGTGAGACA AGCACCAGAAAGACTAGGGTCACCGAGCGAGCCATG CTAGCATG-3'). The SCT DNA was amplified in E. coli DH5 $\alpha$ and prepared using an endotoxin-free DNA Preparation kit (Qiagen, Valencia, CA, USA) according to the manufacturer's instructions.

Detection of SCT expression by flow cytometric analysis. A293 cells were transfected with the corresponding recombinant plasmid DNA by Lipofectamine ${ }^{\mathrm{TM}} 2000$ (Invitrogen) according to the manufacturer's instructions. After $36 \mathrm{~h}$, the transfected cells were collected and stained with FITC-conjugated rat anti-mouse $\mathrm{H}-2 \mathrm{D}^{\mathrm{b}}$ monoclonal antibody (BD Pharmingen, San Diego, CA, USA). Flow cytometric analysis was performed using Becton-Dickinson's FACSCan. 


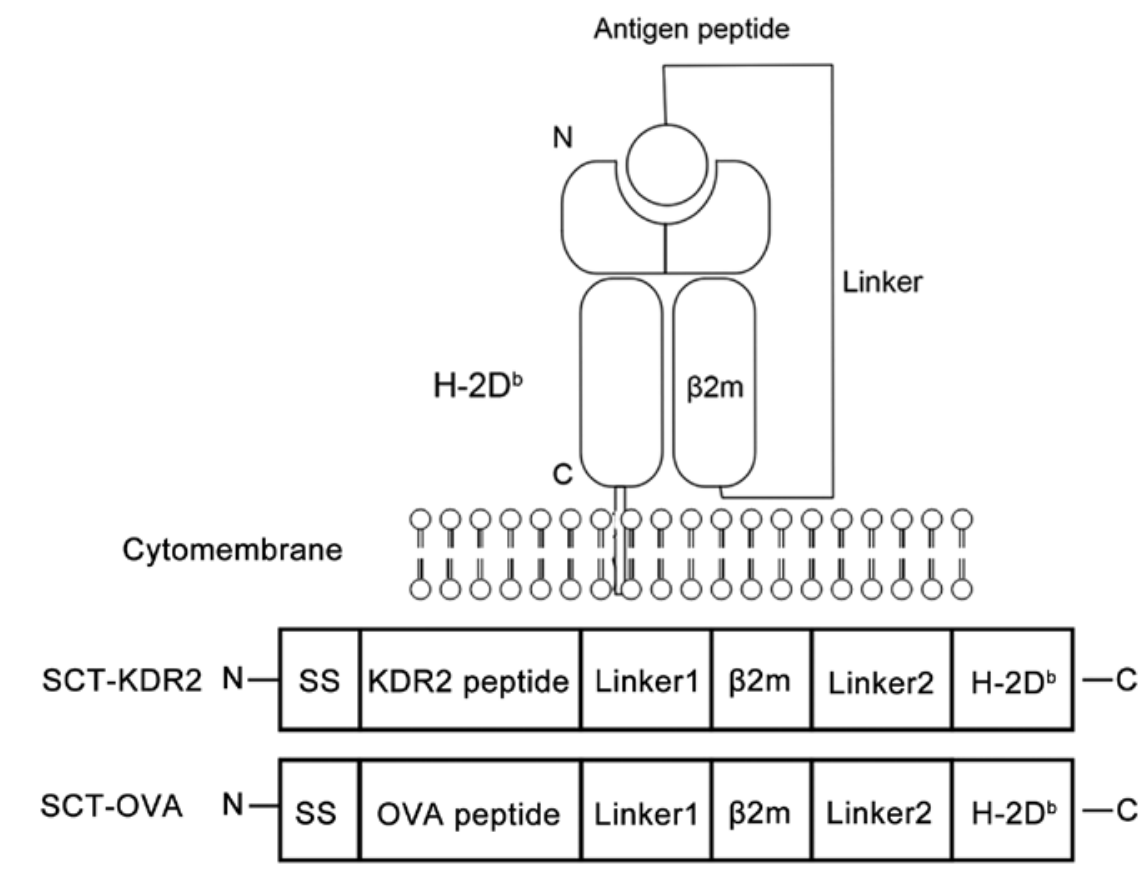

Figure 1. Schematic diagram of SCT constructs. Diagram of an antigen peptide- $\beta 2 \mathrm{~m}-\mathrm{MHC}$ I SCT on cell surface (upper) and diagram of an SCT encoding KDR2 or OVA peptide linked to $\beta 2 \mathrm{~m}$ and $\mathrm{H}-2 \mathrm{D}^{\mathrm{b}}$ (bottom). Each was cloned into pcDNA3.1(+) vector to create the DNA vaccines. SCT, single-chain trimer; $\beta 2 \mathrm{~m}, \beta 2$-microglobulin; OVA, ovalbumin.

Immunization of mice with DNA vaccines. C57BL/6 mice were intradermally immunized three times, at 7-day intervals, with $50 \mu \mathrm{g}$ of pcDNA3.1(+)-KDR2- $\beta 2 \mathrm{~m}-\mathrm{H}-2 \mathrm{D}^{\mathrm{b}}$ (SCT-KDR2), pcDNA3.1(+)-OVA- $\beta 2 \mathrm{~m}-\mathrm{H}-2 \mathrm{D}^{\mathrm{b}}$ (SCT-OVA), or the control DNA pcDNA3.1(+) (vector), respectively. Ten days after the last immunization, the immunized mice were used for evaluation of CTL response to VEGFR2, analyses of tumor cell-induced angiogenesis, or observation of the anti-metastatic effects in murine-metastatic models of B16 melanoma and 3LL Lewis lung carcinoma.

Detection of VEGFR2-specific CTL cytotoxicity. CTL responses were detected as previously described $(20,32)$ with minor modifications. Briefly, 10 days after the last immunization, erythrocyte-depleted splenocytes from mice (n=3-5 per group) were prepared and restimulated for 7 days with mitomycin-inactivated VEGFR2-expressing H5V cell line (at a responder to stimulator ratio of 10:1) in the presence of $50 \mathrm{U} / \mathrm{ml}$ murine IL-2 (PeproTech, UK) in RPMI-1640 with $10 \%$ fetal calf serum. CTL activity was determined by a lactate dehydrogenase (LDH) release assay with CytoTox 96 Non-Radioactive Cytotoxicity Assay kit (Promega, Madison, WI, USA) at an effector:target ratio of 12.5:1, 25:1 and 50:1, respectively. The VEGFR2 ${ }^{+} \mathrm{H} 5 \mathrm{~V}$ and $3 \mathrm{LL}-\mathrm{sflk} 1$ cell lines, and VEGFR2- EL-4 and 3LL cell lines served as the targets, respectively. Specific cytotoxic activity was calculated according to the following: $\%$ specific lysis $=100 \mathrm{x}$ (experimental-effector spontaneous-target spontaneous)/(target maximum-target spontaneous).

Alginate bead analysis of tumor cell-induced angiogenesis. Alginate bead analysis of tumor cell-induced in vivo angiogenesis was performed as previously described (20). Briefly, 3LL Lewis lung carcinoma cells were suspended in $1.5 \%$ sodium alginate and added drop by drop into a swirling $37^{\circ} \mathrm{C}$ solution of $250 \mathrm{mM}$ calcium chloride. Alginate beads (each containing $5 \times 10^{4}$ tumor cells) were prepared. Four beads were implanted subcutaneously into each mouse through an incision made on the dorsal side under anesthetization. After 12 days, $100 \mu 1$ FITC-dextran solution $(20 \mathrm{mg} / \mathrm{ml})$ were intravenously injected into each mouse. The mice were sacrificed 20 min later and alginate beads in each mouse were collected and incubated overnight at room temperature in $1 \mathrm{ml}$ Tris- $\mathrm{HCl}(1 \mathrm{mM}, \mathrm{pH}$ 8.0). The beads were ground with a hand-held mixer and an additional $1 \mathrm{ml}$ Tris- $\mathrm{HCl}(1 \mathrm{mM}, \mathrm{pH} 8.0)$ was added. Samples were vortexed and centrifuged at $250 \mathrm{x}$ g for $5 \mathrm{~min}$. Fluorescence of FITC-dextran in the sample supernatants was measured on a fluorescence spectrophotometer by excitation at $492 \mathrm{~nm}$ and emission at $515 \mathrm{~nm}$. Concentrations of FITCdextran were extrapolated from a standard curve and data were expressed as ng/bead.

Immunohistochemical analysis of tumor-induced angiogenesis. The C57BL/6 mice (5 per group) were intradermally vaccinated three times, at 7-day intervals, with SCT-KDR2, SCT-OVA, or the vector. Ten days after the last immunization, each of the immunized mice was inoculated subcutaneously with $1 \times 10^{5}$ B16 melanoma cells in the rear leg. After 10 days, tumors were removed and fixed in $10 \%$ formalin. Tumors were embedded in paraffin wax, and $5-\mu \mathrm{m}$ sections were cut and used in the immunohistochemical analysis of the factor VIII-related antigen by streptoavidin biotin-peroxidase complex (SABC) method to evaluate tumor-induced angiogenesis as previously described (33), with minor modifications. Briefly, after deparaffinization, the tissue sections were incubated in $0.3 \% \mathrm{H}_{2} \mathrm{O}_{2}$ in methanol for $30 \mathrm{~min}$ to inactivate endogenous peroxidase, and washed with PBS (0.01 M, pH 7.4). The slides then were incubated at $4{ }^{\circ} \mathrm{C}$ overnight with rabbit polyclonal antibody against 
factor VIII-related antigen (1:200; Cell Marque, Rocklin, CA, USA). After washing with TBST (pH7.6), biotinylated goat anti-rabbit IgG (1:1,000; Jackson ImmunoResearch, PA, USA) were applied to the sections for $30 \mathrm{~min}$ at room temperature. The sections were then incubated with streptavidin-HRP (Sigma-Aldrich, St. Louis, MO, USA) for $30 \mathrm{~min}$ at room temperature. HRP activity, which resulted in a brown staining of the reaction sites, was visualized by incubation for $10 \mathrm{~min}$ in Tris-buffered saline containing $0.05 \%$ diaminobenzidine and $0.01 \% \mathrm{H}_{2} \mathrm{O}_{2}$. Hematoxylin was used for the counterstaining.

B16 melanoma metastasis mouse model. C57BL/6 mice (10 per group) were intradermally vaccinated three times, at 7-day intervals, with $50 \mu \mathrm{g}$ SCT-KDR2, SCT-OVA, or vector, respectively. Ten days after the last immunization, the mice were intravenously injected with $1 \times 10^{6} \mathrm{~B} 16$ melanoma cells and were sacrificed 28 days later. Tumor load was evaluated by counting tumor nodules on the lung surfaces as previously described (20). Images of one representative tumor-bearing lung in each group were captured.

3LL Lewis lung carcinoma metastasis mouse model. C57BL/6 mice (10 per group) were intradermally vaccinated three times, at 7-day intervals, with $50 \mu \mathrm{g} \mathrm{SCT-KDR2,} \mathrm{SCT-OVA,} \mathrm{or} \mathrm{vector,}$ respectively. Ten days after the last immunization, the mice received an intrafootpad injection with $2 \times 10^{5} 3 \mathrm{LL}$ tumor cells. When the tumor reached $\sim 6 \mathrm{~mm}$ in diameter, the tumor-bearing leg was amputated surgically. The mice were sacrificed based on the metastatic death in the control groups. Tumor load was evaluated by counting the tumor nodules on the lung surfaces.

Statistical analyses. Statistical analyses were performed using the Student's t-test. $\mathrm{P}<0.05$ was considered statistically significant.

\section{Results}

Expression of KDR2- $\beta 2 m-H-2 D^{b}$ and OVA- $\beta 2 m-H-2 D^{b} S C T$. To characterize the SCT protein expression, human A293 cells were transfected with vector, SCT-KDR2 and SCT-OVA constructs, respectively. SCT expression on transfected cells was examined by flow cytometry with FITC-conjugated rat anti-mouse $\mathrm{H} 2-\mathrm{D}^{\mathrm{b}}$ monoclonal antibody. As shown in Fig. 2, A293 cells transfected with SCT-KDR2 or SCT-OVA expressed mouse $\mathrm{H}-2 \mathrm{D}^{\mathrm{b}}$, whereas A293 cells transfected with vector did not express mouse $\mathrm{H}-2 \mathrm{D}^{\mathrm{b}}$. These results confirmed that the SCT-encoded recombinant plasmids pcDNA3.1(+)KDR2- $\beta 2 \mathrm{~m}-\mathrm{H}-2 \mathrm{D}^{\mathrm{b}}$ and pcDNA3.1(+)-OVA- $\beta 2 \mathrm{~m}-\mathrm{H}-2 \mathrm{D}^{\mathrm{b}}$ could be expressed successfully in eukaryotic cells.

Immunization of mice with DNA vaccine encoding KDR2- $\beta 2 m-H-2 D b$ SCT induces CTL response to VEGFR2. To examine whether vaccination with SCT-KDR2 was able to break self-tolerance and induce CTL response to VEGFR2, mice were intradermally immunized three times, at 7-day intervals, with vector, SCT-OVA and SCT-KDR2. Ten days after the last immunization, splenocytes were prepared and restimulated in vitro for 7 days with the inactivated VEGFR2 ${ }^{+}$ endothelial H5V cell line. CTL activity against H5V (Fig. 3A) and 3LL-sflk1 (Fig. 3B) was determined by LDH release assay.

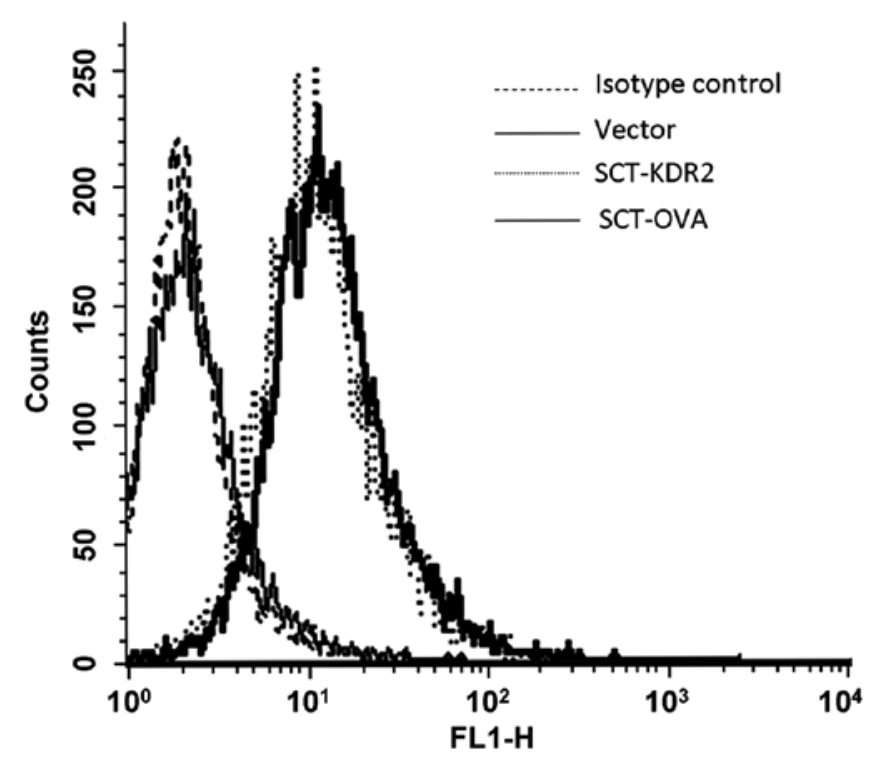

Figure 2. SCT expression in eukaryotic cells. A293 cells were transfected with SCT-KDR2, SCT-OVA, and the vector DNA, respectively. SCT expression on transfected cells was examined by flow cytometry with FITC-conjugated rat anti-mouse $\mathrm{H} 2-\mathrm{D}^{\mathrm{b}}$ monoclonal antibody. SCT, single-chain trimer; OVA, ovalbumin.

As shown in Fig. 3, vaccination of mice with SCT-KDR2 induced CTL responses to VEGFR2 significantly, while, vaccination with vector or SCT-OVA did not induce such responses. Moreover, when the syngeneic VEGFR2-3LL and EL-4 cell lines were used as targets, no significant cytotoxicity was detected (data not shown), suggesting that this CTL response was VEGFR2-specific. Therefore, our data suggested that vaccination of mice with SCT-KDR2 was able to break self-tolerance and induce a CTL response to VEGFR2.

Immunization of mice with DNA vaccine encoding KDR2- $\beta 2 m-H-2 D^{b} S C T$ inhibits tumor-induced angiogenesis. To examine whether the CTL response to VEGFR2 in mice vaccinated with SCT-KDR2 construct inhibited tumor-induced neovascularization, alginate bead and immunohistochemical analyses of tumor cell-induced angiogenesis were performed. As shown in Fig. 4A, FITC-dextran concentration in alginate beads derived from mice immunized with SCT-KDR2 was significantly reduced when compared with that in beads derived from mice vaccinated with the vector or SCT-OVA $(\mathrm{P}<0.01)$. This inhibition reached $53.2 \%$ when compared with the group of SCT-OVA ( $3.6 \pm 0.8$ vs. $7.7 \pm 1.4 \mathrm{ng} /$ bead). Furthermore, the immunohistochemical analysis of factor VIII-related antigen in B16 melanoma showed again that vaccination of mice with SCT-KDR2 inhibited tumorinduced vascularization. As shown in Fig. 4B, tumors in the SCT-KDR group showed markedly decreased factor VIII-related antigen-positive cells compared with those in the vector and SCT-OVA groups. Taken together, our data demonstrated that vaccination of the mice with SCT-KDR2 significantly inhibited tumor cell-induced angiogenesis.

KDR2- $\beta 2 m-H-2 D^{b}$ SCT DNA vaccination inhibits tumor metastasis. To examine the anti-metastatic effects of 
A

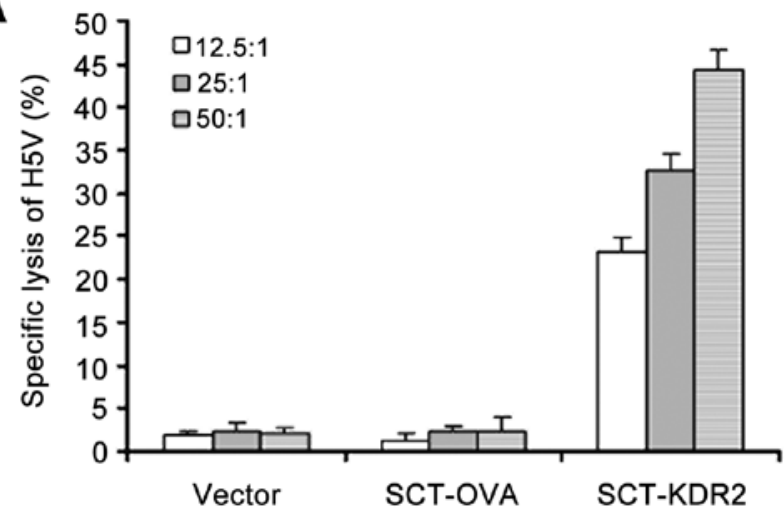

B

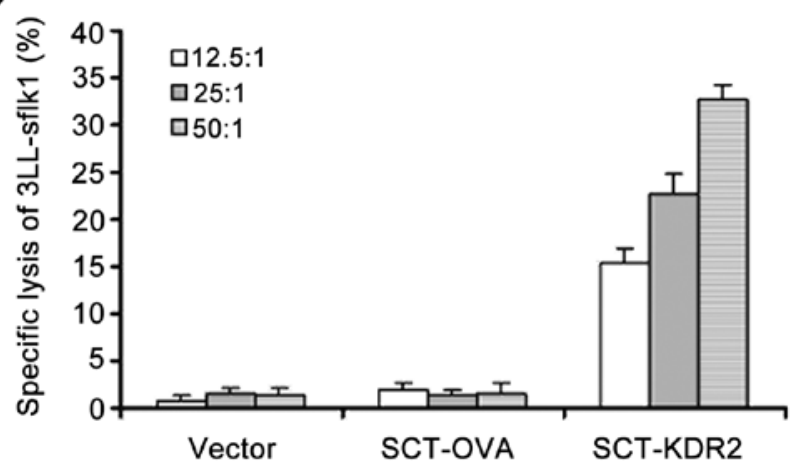

Figure 3. Vaccination of mice with SCT-KDR2 induced CTL response to VEGFR2. CTL activity against (A) H5V and (B) 3LL-sflk1 was determined by LDH release assay at an effector:target ratio of 12.5:1, 25:1 and 50:1, respectively. SCT, single-chain trimer; VEGFR2, vascular endothelial growth factor receptor 2; CTL, cytotoxic T lymphocyte; LDH, lactate dehydrogenase; flk, fetal liver kinase 1 .

SCT-KDR2 vaccination, a murine metastatic model with B16 melanoma was initially employed. As shown in Fig. 5A, mice vaccinated with vector, or SCT-OVA developed extensive pulmonary metastases. However, a marked reduction in pulmonary metastases was observed in mice vaccinated with SCT-KDR2. The number of tumor nodules on lung surfaces decreased by 63.6 and $60 \%$ in mice vaccinated with SCT-KDR2 when compared with that in mice vaccinated with vector, or SCT-OVA, respectively. Moreover, it was obvious that the volumes of the tumor nodules on the lung surfaces from the mice immunized with SCT-KDR2 were significantly reduced in size as compared to those from the mice vaccinated with vector or SCT-OVA (Fig. 5A), which is in agreement with the results of tumor-induced neovascularization. To confirm this finding, anti-metastatic effects using other tumor models, such as the mouse 3LL Lewis lung carcinoma metastatic model, was employed. As shown in Fig. 5B, our data showed again that vaccination of mice with SCT-KDR2 markedly inhibited pulmonary metastasis.

\section{Discussion}

Anti-angiogenic therapy for various types of cancer has attracted much attention in recent years. Blockade of VEGF-A signaling with bevacizumab, a humanized anti-VEGF mono- clonal antibody, or RTKIs has improved progression-free survival and, in some cases overall survival, across many types of cancer, including metastatic colorectal and non-small cell lung cancer, renal cell carcinoma, metastatic breast cancer, and hepatocarcinoma $(6,34)$. However, the clinical benefit of these therapies is variable, and tumors from some treated patients eventually reinitiate growth due to the induction of compensatory angiogenic pathways (35). In a recent study by Croci et al found that glycosylation-dependent lectin-receptor interactions compensate for the absence of cognate ligand and preserve angiogenesis in response to VEGF blockade (7). Remodeling of glycome on the surfaces of endothelial cells regulated the selective binding of Gal1, which following the recognition of complex $\mathrm{N}$-glycans on VEGFR2, activated VEGF-like signaling (7). These findings demonstrated again that VEGFR2-mediated signaling is the rate-limiting step in angiogenesis and VEGFR2 is the most important target for anti-angiogenic therapy. Moreover, in comparison with the administration of angiogenic inhibitors and anti-angiogenic antibodies, anti-angiogenic active immunotherapy has obvious advantages. If a break of immunological tolerance to angiogenesis-promoting regulators is successfully induced, the long-lasting immune response to angiogenesis-related molecule may be present in the body, thereby providing longlasting inhibitory effects on angiogenesis. Therefore, it is expected to be the more cost-effective strategy as compared to angiogenic inhibitor or anti-angiogenic antibody therapy where continuous use of the drugs is required (2).

DNA vaccines have become potentially important therapeutic agents for combating various types of cancer, including anti-angiogenic immunotherapy for cancers, because of their stability, safety, and simplicity (24). Unlike viral or bacterial vectors, DNA vaccines may be administered repeatedly without eliciting a neutralizing antibody response against the vectors themselves. Although DNA vaccines have these advantages, one major drawback is their limited potency, because naked DNA does not have the inherent ability to replicate in vivo. Therefore, attempts have been made to improve the potency of DNA vaccines (24).

It has been proven that a particularly powerful strategy to enhance the potency of DNA vaccines involves bypassing the antigen-processing pathway in DNA-transfected antigenpresenting cells (APCs) by expressing an SCT consisting of an immunogenic peptide, $\beta 2 \mathrm{~m}$ and MHC class I heavy chain $(22,27,36,37)$. The SCT localizes to the plasma membrane of APCs and stably displays an antigenic peptide for recognition by $\mathrm{CD}^{+}$CTLs (22). Therefore, in the present study, we have introduced the SCT platform into our DNA vaccine that consists of KDR2, $\beta 2 \mathrm{~m}$ and $\mathrm{H}-2 \mathrm{D}^{\mathrm{b}}$ and examined its ability to induce CTL response to VEGFR2 and its capacity to inhibit tumor-induced angiogenesis and metastasis in mouse models.

The results show that the constructed SCT DNA vaccine was efficiently expressed in eukaryotic cells. Human A293 kidney embryonic cells were transfected with SCT DNA, and expression of the SCT protein on the cell surface was examined by flow cytometry with FITC-conjugated rat anti-mouse H2-D ${ }^{b}$ monoclonal antibody. As A293 cells do not express mouse MHC class I molecules $\mathrm{H}-2 \mathrm{D}^{\mathrm{b}}$ themselves, the presence of $\mathrm{H}-2 \mathrm{D}^{\mathrm{b}}$ on A293 cells demonstrates that SCT DNA could be successfully expressed. As the main purpose of anti-angiogenic 


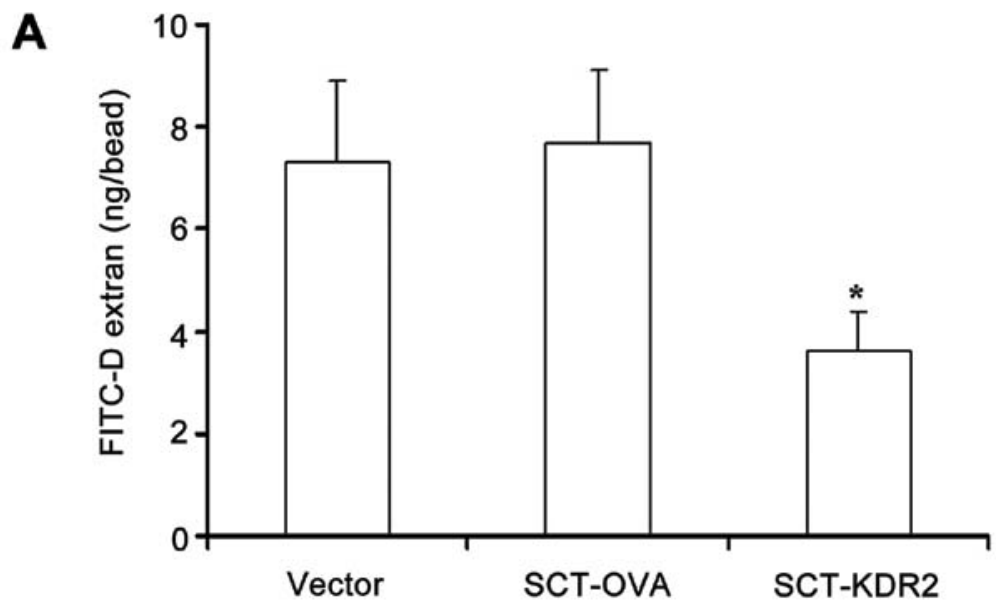

B

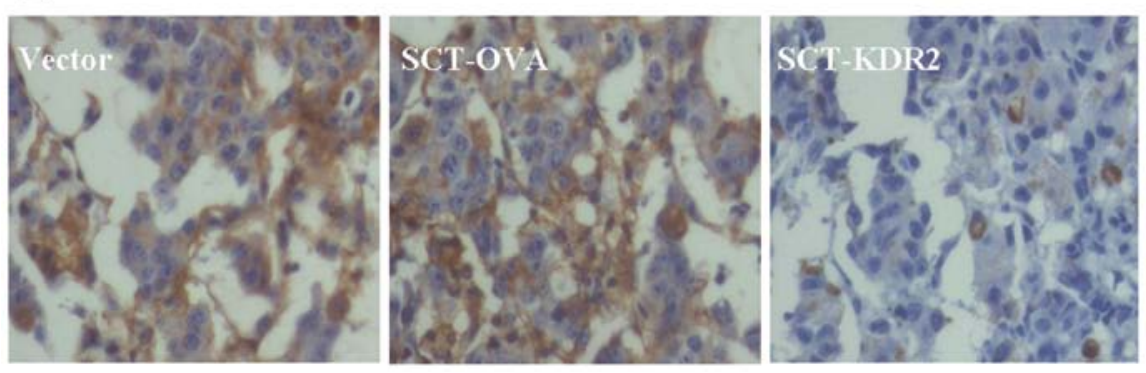

Figure 4. Immunization of mice with DNA vaccine encoding KDR2- $\beta 2 \mathrm{~m}-\mathrm{H}-2 \mathrm{D}^{\mathrm{b}} \mathrm{SCT}$ inhibited tumor cell-induced angiogenesis. (A) Alginate bead analysis of tumor cell-induced angiogenesis. $n=8-10,{ }^{*} \mathrm{P}<0.01$ compared with that in the groups of vector and SCT-OVA. (B) Immunohistochemical analysis of tumor-induced angiogenesis. Original magnification, x200. $\beta 2 \mathrm{~m}, \beta 2$-microglobulin; SCT, single-chain trimer; OVA, ovalbumin.

A
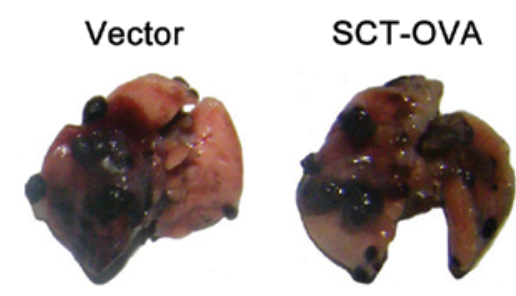

B

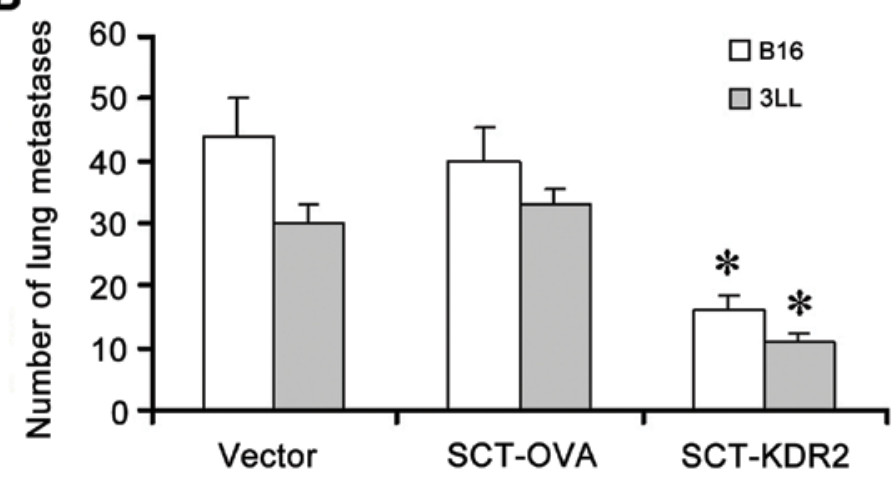

Figure 5. Vaccination of mice with KDR2- $\beta 2 \mathrm{~m}-\mathrm{H}-2 \mathrm{D}^{\mathrm{b}} \mathrm{SCT}$ inhibited tumor metastasis. (A) Vaccination of mice with SCT-KDR2 inhibited tumor metastasis in mouse B16 melanoma model. Images of one representative tumor-bearing lung in each group were shown. (B) Number of tumor nodules on the surfaces of lungs in mouse metastatic models of B16 melanoma or $3 \mathrm{LL}$ Lewis lung carcinoma. ${ }^{\mathrm{P}}<0.01$ when compared with the group of SCT-OVA (n=10). $\beta 2 \mathrm{~m}$, $\beta 2$-microglobulin; SCT, single-chain trimer; OVA, ovalbumin.

active immunotherapy is to break self-immunological tolerance to angiogenesis-promoting regulators and VEGFR2 is the most important therapeutic target in anti-angiogenic therapy for cancers (2), it is critical to determine whether SCT-KDR2 immunization could induce CTL response against VEGFR2. Our results show that CTLs derived from mice vaccinated with 
SCT-KDR2 were able to kill VEGFR2 ${ }^{+}$H5V and 3LL-sflk1, while CTLs from mice vaccinated with vector or SCT-OVA did not kill VEGFR2 ${ }^{+}$H5V and 3LL-sflk1. Furthermore, when EL-4 and 3LL, which are syngeneic VEGFR2- cell lines were used as targets, no significant cytotoxicity was observed, demonstrating that this CTL response was VEGFR2-specific. Therefore, our findings show that vaccination of mice with SCT-KDR2 was able to break self-tolerance and induce CTL response to VEGFR2. We also examined the effects of SCT-KDR2 vaccination on tumor-induced angiogenesis. In accordance with the results in CTL activities, the alginate bead and immunohistochemical analyses showed that vaccination of mice with SCT-KDR2 markedly inhibited tumor-induced angiogenesis. In the alginate bead analysis this inhibition reached $53.2 \%$ when compared with the group vaccinated with SCT-OVA. With regard to the anti-metastatic effects, the results show that the number of metastatic tumors on the lung surfaces in the B16 melanoma and 3LL Lewis lung carcinoma metastatic mouse models vaccinated with SCT-KDR2 markedly decreased when compared with those in mouse models vaccinated with vector or SCT-OVA. As VEGFR2 is strictly expressed in proliferating endothelial cells $(2,38)$, but not in 3LL Lewis lung carcinoma and B16 melanoma cells (20), the anti-metastatic effects observed were considered to be caused indirectly by interference with the tumor cell-induced angiogenesis, which is also supported by the results of the CTL response to VEGFR2 and tumor cell-induced angiogenesis in vivo. Furthermore, the volumes of tumor nodules on the lung surfaces from mice vaccinated with SCT-KDR2 were markedly reduced in size compared to those in mice vaccinated with vector or SCT-OVA, which supports the hypothesis that angiogenesis is critical for tumor outgrowth, progression and metastasis.

Taken together, we have successfully shown that vaccination with SCT-KDR2 DNA was able to break self-immunological tolerance and induce a robust CTL response to VEGFR2, leading to the significant inhibition of metastasis through inhibition of tumor cell-induced angiogenesis in mouse B16 melanoma and 3LL Lewis lung carcinoma metastatic models. Our data show that VEGFR2-targeted SCT vaccination is an effective modality in anti-angiogenic active immunotherapy for various types of cancer and it is important to evaluate this modality further in translational medicine.

\section{Acknowledgements}

This study was supported by grants from the National Natural Science Foundation of China (no. 30872325) and from the Science and Technology Bureau of Hangzhou, Zhejiang Province, P.R. China (no. 20120633B30).

\section{References}

1. Ferrara $\mathrm{N}$ and Kerbel RS: Angiogenesis as a therapeutic target. Nature 438: 967-974, 2005.

2. Pan J, Jin P, Yan J and Kabelitz D: Anti-angiogenic active immunotherapy: a new approach to cancer treatment. Cancer Immunol Immunother 57: 1105-1114, 2008.

3. Chung AS and Ferrara N: Developmental and pathological angiogenesis. Annu Rev Cell Dev Biol 27: 563-584, 2011.

4. Potente M, Gerhardt H and Carmeliet P: Basic and therapeutic aspects of angiogenesis. Cell 146: 873-887, 2011.
5. Yang X, Zhang XF, Lu X, Jia HL, Liang L, Dong QZ, Ye QH and Qin LX: MicroRNA-26a suppresses angiogenesis in human hepatocellular carcinoma by targeting hepatocyte growth factor-cMet pathway. Hepatology 59: 1874-1885, 2014.

6. Shojaei F: Anti-angiogenesis therapy in cancer: Current challenges and future perspectives. Cancer Lett 320: 130-137, 2012.

7. Croci DO, Cerliani JP, Dalotto-Moreno T, et al: Glycosylationdependent lectin-receptor interactions preserve angiogenesis in anti-VEGF refractory tumors. Cell 156: 744-758, 2014.

8. Astorgues-Xerri L, Riveiro ME, Tijeras-Raballand A, Serova M, Neuzillet C, Albert S, Raymond E and Faivre S: Unraveling galectin-1 as a novel therapeutic target for cancer. Cancer Treat Rev 40: 307-319, 2014.

9. Cross MJ and Claesson-Welsh L: FGF and VEGF function in angiogenesis: signalling pathways, biological responses and therapeutic inhibition. Trends Pharmacol Sci 22: 201-207, 2001.

10. Hurwitz H, Fehrenbacher L, Novotny W, et al: Bevacizumab plus irinotecan, fluorouracil, and leucovorin for metastatic colorectal cancer. N Engl J Med 350: 2335-2342, 2004.

11. Sandler A, Gray R, Perry MC, Brahmer J, Schiller JH, Dowlati A, Lilenbaum R and Johnson DH: Paclitaxel-carboplatin alone or with bevacizumab for non-small-cell lung cancer. N Engl J Med 355: 2542-2550, 2006.

12. Miller K, Wang M, Gralow J, Dickler M, Cobleigh M, Perez EA, Shenkier T, Cella D and Davidson NE: Paclitaxel plus bevacizumab versus paclitaxel alone for metastatic breast cancer. N Engl J Med 357: 2666-2676, 2007.

13. Ren S, Fengyu, Zuo S, Zhao M, Wang X, Wang X, Chen Y, Wu Z and Ren Z: Inhibition of tumor angiogenesis in lung cancer by T4 phage surface displaying mVEGFR2 vaccine. Vaccine 29: 5802-5811, 2011.

14. Bracarda S, Caserta C, Sordini L, Rossi M, Hamzay A and Crinò L: Protein kinase inhibitors in the treatment of renal cell carcinoma: Sorafenib. Ann Oncol 18 (Suppl 6): vi22-vi25, 2007.

15. Wei YQ, Wang QR, Zhao X, et al: Immunotherapy of tumors with xenogeneic endothelial cells as a vaccine. Nat Med 6: 1160-1166, 2000.

16. Seavey MM, Maciag PC, Al-Rawi N, Sewell D and Paterson Y: An anti-vascular endothelial growth factor receptor $2 /$ fetal liver kinase-1 Listeria monocytogenes anti-angiogenesis cancer vaccine for the treatment of primary and metastatic Her- $2 / \mathrm{neu}^{+}$breast tumors in a mouse model. J Immunol 182: 5537-5546, 2009.

17. Zuo SG, Chen Y, Wu ZP, et al: Orally administered DNA vaccine delivery by attenuated Salmonella typhimurium targeting fetal liver kinase 1 inhibits murine Lewis lung carcinoma growth and metastasis. Biol Pharm Bull 33: 174-182, 2010.

18. Li Y, Wang MN, Li H, King KD, Bassi R, Sun H, Santiago A, Hooper AT, Bohlen P and Hicklin DJ: Active immunization against the vascular endothelial growth factor receptor flk1 inhibits tumor angiogenesis and metastasis. J Exp Med 195: 1575-1584, 2002.

19. Nair S, Boczkowski D, Moeller B, Dewhirst M, Vieweg J and Gilboa E: Synergy between tumor immunotherapy and antiangiogenic therapy. Blood 102: 964-971, 2003.

20. Pan J, Heiser A, Marget M, Steinmann J and Kabelitz D: Enhanced antimetastatic effect of fetal liver kinase 1 extracellular domain and interferon-gamma fusion gene-modified dendritic cell vaccination. Gene Ther 12: 742-750, 2005.

21. Dong Y, Qian J, Ibrahim R, Berzofsky JA and Khleif SN: Identification of $\mathrm{H}-2 \mathrm{Db}$-specific $\mathrm{CD} 8+\mathrm{T}$-cell epitopes from mouse VEGFR2 that can inhibit angiogenesis and tumor growth. J Immunother 29: 32-40, 2006.

22. Hansen T, Yu YY and Fremont DH: Preparation of stable singlechain trimers engineered with peptide, beta 2 microglobulin, and MHC heavy chain. Curr Protoc Immunol Chapter 17: Unit 17.15, 2009.

23. Huang CH, Peng S, He L, Tsai YC, Boyd DA, Hansen TH, Wu TC and Hung CF: Cancer immunotherapy using a DNA vaccine encoding a single-chain trimer of MHC class I linked to an HPV-16 E6 immunodominant CTL epitope. Gene Ther 12: 1180-1186, 2005.

24. Kang TH, Mao CP, La V, Chen A, Hung CF and Wu TC: Innovative DNA vaccine to break immune tolerance against tumor self-antigen. Hum Gene Ther 24: 181-188, 2013.

25. Hung CF, Calizo R, Tsai YC, He L and Wu TC: A DNA vaccine encoding a single-chain trimer of HLA-A2 linked to human mesothelin peptide generates anti-tumor effects against human mesothelin-expressing tumors. Vaccine 25: 127-135, 2007.

26. Huang B, Mao CP, Peng S, He L, Hung CF and Wu TC: Intradermal administration of DNA vaccines combining a strategy to bypass antigen processing with a strategy to prolong dendritic cell survival enhances DNA vaccine potency. Vaccine 25: 7824-7831, 2007. 
27. Kim S, Zuiani A, Carrero JA and Hansen TH: Single chain MHC I trimer-based DNA vaccines for protection against Listeria monocytogenes infection. Vaccine 30: 2178-2186, 2012.

28. Kim S, Li L, McMurtrey CP, Hildebrand WH, Weidanz JA, Gillanders WE, Diamond MS and Hansen TH: Single-chain HLA-A2 MHC trimers that incorporate an immundominant peptide elicit protective $\mathrm{T}$ cell immunity against lethal West Nile virus infection. J Immunol 184: 4423-4430, 2010.

29. Cheung YK, Cheng SC, Ke Y and Xie Y: Two novel HLA-A*0201 T-cell epitopes in avian H5N1 viral nucleoprotein induced specific immune responses in HHD mice. Vet Res 41: 24, 2010.

30. Cheung YK, Cheng SC, Sin FW, Chan KT and Xie Y: Investigation of immunogenic T-cell epitopes in SARS virus nucleocapsid protein and their role in the prevention and treatment of SARS infection. Hong Kong Med J 14: (Suppl 4): 27-30, 2008.

31. Cheung YK, Cheng SC, Sin FW, Chan KT and Xie Y: Induction of T-cell response by a DNA vaccine encoding a novel HLA-A*0201 severe acute respiratory syndrome coronavirus epitope. Vaccine 25: 6070-6077, 2007.

32. Hu YX, Li M, Jia XH, Du QX, Miao FT, Yao L and Shen JD: HPV16 CTL epitope peptide-activated dendritic cell and natural killer co-culture for therapy of cervical cancer in an animal model. Asian Pac J Cancer Prev 14: 7335-7338, 2013.
33. Wang D, Stockard CR, Harkins L, et al: Immunohistochemistry in the evaluation of neovascularization in tumor xenografts. Biotech Histochem 83: 179-189, 2008.

34. Ellis LM and Hicklin DJ: VEGF-targeted therapy: Mechanisms of anti-tumour activity. Nat Rev Cancer 8: 579-591, 2008.

35. Ebos JM, Lee CR and Kerbel RS: Tumor and host-mediated pathways of resistance and disease progression in response to antiangiogenic therapy. Clin Cancer Res 15: 5020-5025, 2009.

36. Yu YY, Netuschil N, Lybarger L, Connolly JM and Hansen TH: Cutting edge: Single-chain trimers of MHC class I molecules form stable structures that potently stimulate antigen-specific $\mathrm{T}$ cells and B cells. J Immunol 168: 3145-3149, 2002.

37. Lybarger L, Yu YY, Miley MJ, Fremont DH, Myers N, Primeau T, Truscott SM, Connolly JM and Hansen TH: Enhanced immune presentation of a single-chain major histocompatibility complex class I molecule engineered to optimize linkage of a C-terminally extended peptide. J Biol Chem 278: 27105-27111, 2003.

38. Kanagawa N, Yanagawa T, Nakagawa T, Okada N and Nakagawa S: Tumor vessel-injuring ability improves antitumor effect of cytotoxic $\mathrm{T}$ lymphocytes in adoptive immunotherapy. Cancer Gene Ther 20: 57-64, 2013. 